\title{
Super-Resolution of the Radio Remnant of SN 1987A
}

B. M. Gaensler ${ }^{1}$, A. K. Tzioumis, R. N. Manchester, L. Staveley-Smith, J. E. Reynolds, \& M. J. Kesteven Australia Telescope National Facility, PO Box 76, Epping, NSW 2121, Australia

Abstract. The radio remnant of $\mathrm{SN} 1987 \mathrm{~A}$ is now of sufficient flux density that superresolution is possible at $9 \mathrm{GHz}$. We present a sequence of images from 1992 to 1996 with a resolution of $\approx 0.0^{\prime \prime} 5$, showing the continuing interaction between the expanding shock front and the surrounding axisymmetric circumstellar material.

\section{Introduction}

After a brief outburst of emission lasting $\sim 6$ months, radio emission was redetected from SN 1987A by both the Molonglo Observatory Synthesis Telescope (MOST) and the Australia Telescope Compact Array (ATCA) in mid-1990, approximately 1200 days after the explosion. This second onset of emission represents the encounter between the expanding supernova shock and the dense red supergiant wind of the progenitor, giving us the unique opportunity to observe the birth of a supernova remnant. Gaensler et al. (1997) discuss these results in detail-a brief update is given here.

\section{Results}

At $9 \mathrm{GHz}$, the resolution of ATCA is $\sim 0.9$, just sufficient to resolve the remnant of SN 1987A. However after combining all the data from a given year, the following criteria are satisfied:

- Good signal-to-noise (integration times of $\sim 50 \mathrm{hr}$ per image)

- Excellent $u, v$ coverage (multiple configurations \& multi-frequency synthesis)

- Relatively simple structure

This allows us to perform super-resolution, where we improve the resolution of our images by using a restoring beam smaller than the diffraction limit. Extensive tests (Briggs 1995) have shown that physically meaningful and reproducible results can be produced for SN $1987 \mathrm{~A}$ when using a Gaussian restoring beam of FWHM 0.4. The results of this technique are shown in Figure 1, the similarity of the images an indicator of the reliability of the deconvolution process.

The radio shell is dominated by two bright lobes, whose position angles align with the major axis of the optical ring seen with $H S T$ (Plait et al. 1995). In terms of the three dimensional geometry of the system inferred from optical observations (Burrows et al. 1995), this morphology is consistent with the radio

\footnotetext{
${ }^{1}$ also Astrophysics Department, School of Physics A29, University of Sydney, NSW 2006,
} Australia 


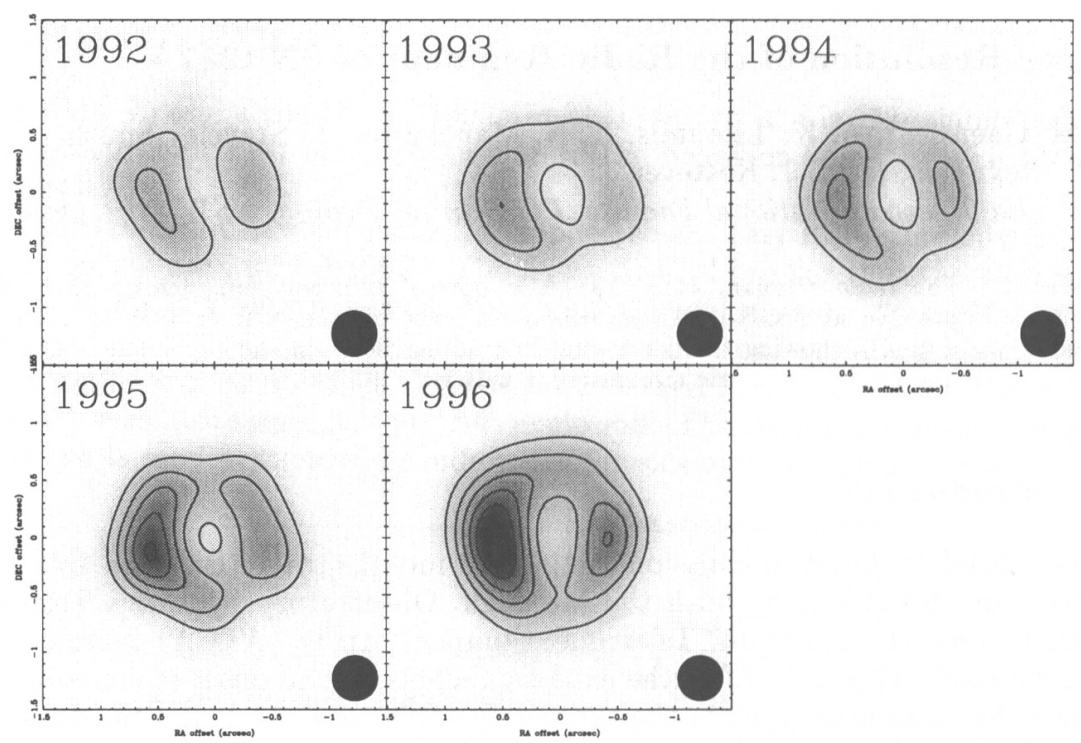

Figure 1. Super-resolved $9 \mathrm{GHz}$ images of SN 1987A from 1992 to 1996. Contour levels are from 0.4 to $2.4 \mathrm{mJy}$ beam ${ }^{-1}$ at $0.4 \mathrm{mJy}^{2} \mathrm{beam}^{-1}$ intervals. At lower right of each image is shown the FWHM 0.4 restoring beam. The offset coordinates are relative to RA (J2000) $05^{\mathrm{h}} 35^{\mathrm{m}} 27^{\mathrm{s}} .97$, Dec (J2000) $-69^{\circ} 16^{\prime} 11^{\prime \prime} 1$.

emitting regions being located in the equatorial plane of the progenitor system. This is in agreement with theoretical models for the circumstellar medium of SN 1987 A (Blondin \& Lundqvist 1993; Chevalier \& Dwarkadas 1995), which predict that the material which the shock has encountered is densest and lies closest to the progenitor in the equatorial plane.

An expansion speed of $2400 \pm 300 \mathrm{~km} \mathrm{~s}^{-1}$ can be calculated from the $(u, v)$ data corresponding to the images in Figure 1. At this rate, the supernova shock will encounter the dense material demarcated by the optical ring in $2008 \pm 3$. When this occurs, the source will become a strong radio, X-ray, optical, IR and UV source for $\sim 30$ years.

Acknowledgments. The Australia Telescope is funded by the Commonwealth of Australia for operation as a National Facility managed by CSIRO.

\section{References}

Blondin, J. M., \& Lundqvist, P. 1993. ApJ, 405, 337-352.

Briggs, D. S. 1995. Ph.D. Thesis, New Mexico Institute of Mining and Technology.

Burrows, C. J., et al. 1995. ApJ, 452, 680-684.

Chevalier, R. A., \& Dwarkadas, V. V. 1995. ApJ, 452, L45-48.

Gaensler, B. M., et al. 1997. ApJ, 479, 845-858.

Plait, P. C., et al. 1995. $A p J, 439,730-751$. 